FORMATION Formation emploi

Revue française de sciences sociales

98 | avril-juin 2007

Pour une approche par les capacités

\title{
Capacités et développement professionnel
}

Capabilities and occupational development

„Capabilities“ und berufliche Fortbildung

Delphine Corteel et Bénédicte Zimmermann

\section{OpenEdition}

Journals

Édition électronique

URL : http://journals.openedition.org/formationemploi/1561

DOI : $10.4000 /$ formationemploi. 1561

ISSN : 2107-0946

Éditeur

La Documentation française

Édition imprimée

Date de publication : 1 avril 2007

Pagination : 25-39

ISSN : 0759-6340

Référence électronique

Delphine Corteel et Bénédicte Zimmermann, «Capacités et développement professionnel », Formation emploi [En ligne], 98 | avril-juin 2007, mis en ligne le 30 juin 2009, consulté le 30 octobre 2020. URL

http://journals.openedition.org/formationemploi/1561 ; DOI : https://doi.org/10.4000/

formationemploi.1561

() Tous droits réservés 


\title{
Numéro spécial
}

\section{Capacités et développement professionnel}

\author{
Par Delphine Corteel et Bénédicte Zimmermann*
}

\begin{abstract}
L'employabilité, la responsabilité individuelle et l'activation des politiques d'emploi sont aujourd'hui les maîtres-mots d'une réforme de l'État social placée sous le signe de la flexicurité. Mais quelles sont les possibilités effectives offertes en entreprise aux salariés en vue de leur développement professionnel?
\end{abstract}

Les directives européennes pour l'emploi ont fait de la notion d'employabilité - capacité d'adaptation professionnelle dans la version française - une pièce maitresse des réformes nationales de la protection sociale (Commission européenne, 2000, p. 3). Elles visent à substituer à une logique curative dite «passive», historiquement portée par le collectif, une logique préventive "active» qui cherche son ancrage dans la personne du travailleur (Barbier, 2002). Il en résulte un déplacement de la responsabilité collective vers la responsabilité individuelle: responsables de leur employabilité, les salariés sont appelés à devenir les entrepreneurs de leur développement professionnel.

Ce déplacement de responsabilité est ambivalent. D'une part, il valorise et encourage la latitude d'action individuelle et la liberté d'accomplissement personnel ; d'autre part, il a pour conséquence d'imputer l'absence d'évolution professionnelle et les causes du chômage de longue durée à la personne même du chômeur. Accordant une place nouvelle à l'idée de liberté et d'initiative individuelle aux côtés de celle de sécurité, ce discours de réforme joue sur la confusion entre deux acceptions de la liberté, d'où son ambivalence: d'une part la liberté «négative», entendue comme l'absence d'entraves à l'action,

" Delphine Corteel, anthropologue, est maîtresse de conférences à l'université de Reims Champagne-Ardenne et membre du Laboratoire analyse et évaluation des professionnalisations. Elle a coordonné le numéro 2001-3 d'Ethnologie Française intitulé "Anthropologie ouvrière et enquête d'usine » (en collaboration avec Sylvain Lazarus) et publié plusieurs textes sur les ouvriers de l'industrie automobile allemande, leur rapport au travail et à l'usine, notamment dans Histoire et Sociétés, 2005, n 13 .

Bénédicte Zimmermann, sociologue, est maîtresse de conférences à l'École des hautes études en sciences sociales à Paris et membre du Centre de recherches interdisciplinaires sur l'Allemagne (CNRS / EHESS). Elle est l'auteure de La constitution du chômage en Allemagne. Entre professions et territoires (Paris, Éd. de la MSH, 2001). Elle a dirigé plusieurs ouvrages dont Le travail et la nation. Histoire croisée de la France et de l'Allemagne (Paris, Éd. de la MSH, 1999, en collaboration avec Claude Didry et Peter Wagner) et Les sciences sociales à l'épreuve de l'action. Le savant, le politique et l'Europe (Paris, Éd. de la MSH, 2004) 
d'autre part la liberté « positive » qui met au premier plan la capacité effective d'action d'une personne dans une situation donnée ${ }^{1}$. Alors que les réformes actuelles sont marquées par la conception « négative » de la liberté qui prévaut dans le libéralisme économique qui les inspire, elles ne font que peu de cas de la liberté «positive». Or A. Sen (2003) attire notre attention sur l'interdépendance entre ces deux conceptions de la liberté. Prenant le contre-pied $\mathrm{du}$ discours économique dominant, il rappelle que la liberté est aussi une valeur du développement humain, associée à l'accomplissement personnel et la reconnaissance de soi. Il montre qu'on ne peut dissocier la prise de responsabilité de l'exercice de la liberté, pas plus qu'on ne peut dissocier l'exercice de la liberté de l'égale distribution des opportunités sociales. En d'autres termes, pour Sen, l'exercice de la responsabilité individuelle requiert une liberté positive d'action que seule une responsabilité sociale peut, dans les faits, garantir à chacun. L'approche par les capacités ${ }^{2}$ développée par Sen amène ainsi à réévaluer les rapports entre efficience économique, responsabilité individuelle et équité sociale à partir de la question de la liberté. Tout en accordant une place centrale à la responsabilité individuelle, elle n'en fait pas pour autant un substitut de la responsabilité collective, mais déplace l'objet de cette dernière de la protection des salariés vers l'égale distribution des possibles et des opportunités sociales.

La liberté a jusqu'à présent été peu thématisée par la sociologie du travail, davantage centrée sur la notion d'autonomie et son envers : la servitude et l'aliénation, dans le prolongement de la critique du salariat et de la société de consommation, respectivement développée par K. Marx et H. Arendt. Penser ensemble la responsabilité, la sécurité et la liberté implique par conséquent la production d'outils intellectuels permettant de faire de la liberté un objet sociologique. Cette exigence suppose de ne pas seulement focaliser l'analyse sur les

\footnotetext{
${ }^{1}$ Sur la distinction entre liberté « négative» et « positive », voir notamment I. Berlin, 1988, pp. 165-218.

${ }^{2}$ Le terme de capacité, qui a en français une connotation juridique, renvoie historiquement aux droits ou aux jouissances associées à un statut. De ce fait, il est mal approprié à restituer l'idée d'une possible mobilité entre les statuts contenue dans la notion anglaise de capability. Du point de vue de la stricte rigueur intellectuelle, il serait par conséquent préférable de recourir en français au néologisme « capabilité », mais ces réserves étant posées, nous nous en tiendrons, pour des facilités de lecture, au terme de capacité.
}

politiques du marché du travail, les instruments et les ressources existantes, mais de prendre en compte également l'accès différentiel à ces ressources, les opportunités de choix des salariés, la nature des compromis qui déterminent l'étendue de ces choix, enfin les réalisations effectives qui en découlent.

Alors que les institutions publiques font désormais de l'employabilité et du développement des compétences le point cardinal de la sécurité des salariés, la prise en compte de la latitude d'action des personnes amène à déplacer l'analyse de la stricte notion de compétences vers celle, plus large, de capacités. La démarche compétences, telle qu'elle est mise en œuvre par les entreprises, tend d'abord à satisfaire des besoins de flexibilité et d'adaptablité et, ainsi, à promouvoir une conception essentiellement instrumentale de l'employabilité. Elle apporte une réponse à l'impératif économique de réactivité et d'ajustement en juste à temps, mais fait peu de place à la dynamique temporelle et sociale des parcours individuels, de même qu'à la liberté de choix personnel (Zimmermann, 2006a). L'approche par les capacités, en revanche, prend en compte à la fois ce qu'une personne est capable de faire - ses compétences - et les possibilités qui lui sont offertes pour développer ces compétences - les opportunités ${ }^{3}$. Elle pose en des termes nouveaux la question de la sécurité et de la responsabilité individuelle en l'arrimant à la question de la liberté et du collectif.

La notion de capacité, telle que nous l'utilisons, implique tout à la fois une latitude de choix individuel, des opportunités réelles, leur conversion en réalisations effectives ${ }^{4}$, un pouvoir d'agir soutenu par des supports collectifs, et enfin la possibilité pour les salariés d'exprimer leurs préférences et de les faire valoir. Cette acception de la notion de capacité s'appuie sur la définition plus générale qu'en donne Sen :

«La liberté de mener différentes sortes de vies correspond exactement à l'ensemble formé par différentes combinaisons de fonctionnements humains,

${ }^{3}$ Selon la distinction opérée par D. Gasper (2002, p. 446) entre skills-capabilities et opportunities-capabilities.

4 À la suite de R. Castel (2000), nous entendons par supports collectifs un ensemble de droits, de ressources matérielles et symboliques prodiguées par des collectifs de différente nature, allant du règlement d'atelier jusqu'aux politiques nationales et européennes en passant par les politiques d'entreprises, l'action syndicale et les dispositifs publics. 
ensemble en lequel une personne est à même de choisir sa vie. C'est ce qu'on peut appeler la "capabilité" de la personne. La "capabilité" d'une personne dépend de nombreux éléments qui comprennent aussi bien les caractéristiques personnelles que l'organisation sociale. La responsabilité de la société à l'égard de la liberté individuelle impose qu'on attache de l'importance à l'accroissement des "capabilités" dont disposent réellement des personnes différentes. » (Sen, 2003, pp. 64-65)

La problématisation des rapports entre travail et liberté amène ainsi à sortir d'une conception strictement économique du travail pour prendre en compte ses interactions avec la vie privée et familiale, avec l'engagement citoyen et participatif dans et hors de l'entreprise, plus largement avec les formes de l'action publique et de l'organisation sociale.

Cet article s'appuie sur les résultats d'une enquête menée entre 2003 et 2005 auprès de huit entreprises françaises de la chimie, de la métallurgie et de trois services municipaux de propreté urbaine. L'enquête traitait de l'impact des politiques d'entreprises sur les parcours individuels et explorait de manière approfondie la question des opportunités sociales et des supports collectifs accessibles aux salariés à des fins de développement professionnel. Après une présentation du dispositif d'enquête, nous étudierons les différentes configurations qui se dégagent des cas étudiés, en prêtant une attention particulière aux conditions de développement des capacités des salariés en entreprise.

\section{ENQUÊTER SUR LES CAPACITÉS}

L'approche par les capacités laisse la question de la méthode ouverte, déléguant au chercheur le soin de définir une méthodologie adaptée. Initialement élaborée dans le cadre d'études sur les pays en voie de développement, elle a généré de nombreux travaux quantitatifs, essentiellement en économie, alors que la sociologie et l'anthropologie s'en sont peu saisies. La réflexion que nous avons menée sur son opérationnalisation empirique dans ces disciplines, sur la façon dont des possibles, des espaces d'opportunités et des choix personnels peuvent empiriquement être saisis et analysés par les sciences sociales, nous a, pour notre part, amenées à privilégier une méthode qualitative ${ }^{5}$.

Ce choix nous a conduit à centrer l'enquête sur les parcours biographiques et les contextes organisationnels, en croisant dimensions institutionnelles, organisationnelles et biographiques ${ }^{6}$.

- Nous ne considérons pas l'entreprise comme une organisation fermée sur elle-même, mais partie prenante d'un secteur d'activité, inscrite dans des réseaux de fournisseurs et de clients, ouverte sur un territoire, en prise avec la concurrence et régulée par des conventions collectives et des lois. Ces données institutionnelles déterminent des ensembles spécifiques de contraintes et de ressources - qualifications, expérience professionnelle, droits formels -, délimitant un espace de parcours possibles dans un territoire et un secteur donnés. Il en résulte une perspective institutionnelle ${ }^{7}$.

- À l'échelle de chaque entreprise, l'enquête a porté sur les produits, le modèle de production et son évolution (Salais et Storper, 1993), de même que sur l'organisation du travail et la politique des ressources humaines. Nous nous sommes particulièrement intéressées aux outils et aux pratiques d'évaluation associés à trois moments-clés susceptibles de constituer des tournants dans les parcours individuels : le recrutement, la formation continue et la mobilité professionnelle. Axée sur les opportunités et les supports collectifs internes qui contribuent à structurer les parcours, cette perspective organisationnelle permet de dégager des configurations communes à plusieurs entreprises.

- Enfin, une troisième perspective, au plus près des personnes, s'appuie sur l'analyse de quelques deux cents récits de salariés de tous niveaux hiérarchiques. Les entretiens mettaient l'accent sur la formation et le parcours professionnel de la personne, ses souhaits et ses projets, sa latitude de choix, les réalisations effectives et les non-réalisations, la conciliation entre

\footnotetext{
${ }^{5}$ Nous ne reviendrons pas ici sur les motifs de ce choix ; pour leur explicitation, voir Zimmermann, 2006b.

${ }^{6} \mathrm{C}$ 'est à dessein que nous utilisons le terme de parcours, plutôt que de trajectoire. La notion de trajectoire, inspirée par le modèle balistique, renvoie, dans la tradition sociologique, à une perspective déterministe (Passeron, 1990, p. 21), peu adaptée à un examen des formes de liberté.

${ }^{7}$ Nous avons nous-même mené une enquête territoriale dans la région des Pays de la Loire (voir Corteel et Zimmermann, 2006). En ce qui concerne la zone industrialo-portuaire du Havre, nous nous sommes appuyées sur les travaux de Zalio et Fouillant, 2004.
} 
le travail salarié, d'autres types d'activités et la vie privée. Cette perspective biographique permet de sortir du strict cadre de l'entreprise. Elle donne accès aux préférences des personnes à un moment particulier de leur vie et à la façon dont elles identifient l'impact des données institutionnelles, organisationnelles et biographiques sur leur propre parcours.
Croiser ces trois perspectives permet de dessiner un faisceau de ressources et de contraintes, de possibilités et d'opportunités, mais également d'impossibilités, dont nous avons cherché à mesurer les effets sur le développement des capacités professionnelles des salariés. Les tournants et les bifurcations de parcours d'une part, les critères d'évaluation et d'accès aux

\section{Encadré 1 \\ L'enquête}

\section{Le Havre - Zone industrialo-portuaire}

Métallurgie $\quad \mathrm{H} 2 \mathrm{O}-$ Nacelles de moteurs d'avion - 1200 salariés - 16 entretiens

Chimie Luz - Additifs pour lubrifiants - 200 salariés -12 entretiens

Herculanum - Producteur de TiO2 -340 salariés -13 entretiens

Helios - Fabricant multi produits - 280 salariés -7 entretiens

Secteur public Propreté urbaine -220 salariés -5 entretiens

\section{Région Pays de la Loire}

Métallurgie Bigtrucks - Assembleur de poids lourds -450 salariés -25 entretiens Matrix - Fabricant de composants électroniques -700 salariés -39 entretiens Watson - Production et réparation d'électroménager - 400 salariés -11 entretiens

Chimie Paintings - fabricant de vernis et peintures - 30 salariés -5 entretiens

Secteur public Propreté urbaine -212 salariés -6 entretiens

Paris

Secteur public Services Techniques de la Propreté de Paris - 4500 salariés - 43 entretiens

Ces enquêtes se sont déroulées d'octobre 2003 à mars 2005. Elles comprenaient des séjours d'observation et des entretiens semi-directifs conduits sur la base d'une grille d'entretien composée de questions ouvertes. Dans chaque entreprise, les entretiens se sont déroulés avec des salariés de différents niveaux hiérarchiques - de l'ouvrier au PDG - ainsi qu'avec des représentants élus du personnel. Les entretiens portaient systématiquement sur le parcours professionnel de la personne rencontrée et sa perception de la politique des ressources humaines; ils comportaient également un volet spécifique en fonction du poste de notre interlocuteur. En outre, tous les documents disponibles - supports de communication, bilan social, référentiels emploi, guides et supports d'évaluation, guides de formation et accords d'entreprises, etc. - ont été collectés et analysés.

Cette recherche s'insère dans un programme collectif européen $\left({ }^{*}\right)$ dont le cahier des charges définissait les secteurs d'investigation : les services régulés par l'État - en l'occurrence, les services municipaux de propreté urbaine -, la métallurgie et la chimie. Les deux derniers secteurs peuvent être considérés comme des secteurs industriels traditionnels. Leur forte présence dans notre échantillon lui confère une tonalité particulière. Les configurations qui en résultent ne prétendent donc pas à la représentativité. Elles permettent toutefois de dégager des caractéristiques organisationnelles et entrepreneuriales plus ou moins favorables à la latitude d'action des salariés en matière de développement professionnel.

De la même manière, l'implantation des entreprises dans deux territoires, les Pays de la Loire et la zone industrialo-portuaire du Havre, a été déterminée par le cahier des charges du programme européen de recherche.

$(*)$ «Eurocap : Dialogue Social, Emploi et Territoire, pour une politique européenne des capacités 》 (http://www.idhe.enscachan.fr/Eurocap/). La grille d'enquête a été élaborée en commun avec les équipes des sept pays impliqués dans le projet. Corteel et Zimmermann, 2006, pp. 137-142. 
ressources dans ces moments clés d'autre part, constituent les deux axes qui structurent notre analyse. En introduisant le changement dans une configuration donnée, les tournants biographiques constituent un puissant révélateur des espaces d'opportunités, alors que les modalités et les critères d'évaluation cristallisent les interactions entre données personnelles, organisationnelles et institutionnelles qui façonnent les épreuves de passage ${ }^{8}$. Ces épreuves se sont profondément transformées au cours des dernières années (Eymard-Duvernay et Marchal, 1997 ; Dejours, 2003 ; Bureau et Marchal, 2005), induisant une pluralisation des critères et un déplacement du centre de gravité de l'évaluation des services du personnel vers les supérieurs hiérarchiques directs $(n+1)$. L'évaluation porte désormais autant sur la réalisation du travail, la qualité du produit ou du service que sur la personne et ses compétences relationnelles, brouillant la lisibilité des épreuves pour les salariés.

Deux grandes configurations se dégagent de l'enquête, en fonction des opportunités et des soutiens que les entreprises offrent aux personnes pour l'exercice de leurs libertés dans le travail et l'emploi. D'une part, des entreprises qui présentent peu d'opportunités de développement professionnel ; parmi celles-ci, il convient de distinguer les entreprises en restructuration qui n'offrent ni opportunités ni sécurité, des entreprises qui, en raison de leur très petite taille ou de la structure particulière des emplois (services municipaux de propreté), offrent peu d'opportunités mais une sécurité relative. D'autre part, des entreprises qui offrent des opportunités de développement, mais dont certaines seulement font de l'évolution professionnelle une responsabilité collective. Selon les cas, nous mettrons plus spécifiquement l'accent sur les critères d'évaluation en matière de recrutement, de formation ou de promotion, en fonction de leur importance respective dans les contextes organisationnels étudiés.

\footnotetext{
${ }^{8}$ Nous entendons la notion d'épreuve au double sens étymologique de ce qui permet de conférer une valeur et de ce qui s'éprouve dans l'adversité et la souffrance. Au sens où nous l'utilisons, l'épreuve renvoie à des dispositifs permettant de classer les individus en fonction de grandeurs et de relations d'équivalence associées à des principes de justice (L. Boltanski et L. Thévenot, 1991), mais aussi à l'incertitude et la mise à l'épreuve de soi.
}

\section{DES PERSPECTIVES DE DÉVELOPPEMENT PROFESSIONNEL LIMITÉES}

L'existence d'opportunités de développement est une première condition nécessaire au déploiement des capacités. Or, plus de la moitié des entreprises de notre échantillon ne remplissent pas, ou très mal, cette condition. En effet, la situation économique des entreprises, leur taille, le degré d'institutionnalisation de la gestion des carrières (cas du service public), mais aussi la politique des ressources humaines jouent un rôle déterminant. Pour les personnes impliquées dans les trois contextes organisationnels que nous allons présenter ci-après, la question de l'exercice de la liberté dans le travail ne se pose quasiment pas. Au regard des ressources dont elles disposent et de l'environnement dans lequel elles évoluent, avoir et conserver son emploi représente l'enjeu fondamental. Selon les contextes, elles bénéficient cependant d'un degré de sécurité variable : très faible dans les entreprises en restructuration, relatif dans les PME et maximal dans les services municipaux de propreté.

\section{Se former... mais pourquoi? Le cas des restructurations}

Notre échantillon comprend trois entreprises en situation de crise : Herculanum, Watson et Matrix, qui a d'ailleurs fermé depuis l'enquête. Ces entreprises relevaient toutes du modèle de production industriel ${ }^{9}$. Afin de juguler la crise, certaines s'orientent vers une diversification des produits et des activités. Herculanum reste fidèle au modèle industriel, mais restructure sa production et investit dans la modernisation de ses installations; Matrix tente de développer un modèle de production de type marchand basé sur une diversification des produits et des clients, avec la fabrication de cartes électroniques ; Watson ajoute un

\footnotetext{
${ }^{9}$ Nous nous référons ici à la typologie des mondes de production de Salais et Storper (1993). Ces auteurs distinguent quatre mondes : industriel (produits standards de masse), marchand (produits standards dédiés), interpersonnel (produits socialisés dédiés), immatériel (recherche et développement, etc.). Notre échantillon reflète une forte évolution des modèles de production industriels vers des modèles marchands ou du moins vers un mixage des modèles au sein d'une même entreprise (Corteel et Zimmermann, 2006, 35 sqq.).
} 
centre de réparation à ses chaînes de production d'électroménager. Ces réorganisations ont donné lieu aux programmes de formation les plus ambitieux que nous ayons pu observer. Ainsi, Matrix et Watson ont confié, l'une à l'AFPI (Association de formation professionnelle de l'industrie), l'autre à l'AFPA (Association nationale pour la formation professionnelle des adultes), le soin d'élaborer des programmes sur mesure, d'une durée de plusieurs semaines, afin de former rapidement quelques dizaines d'ouvriers aux nouveaux produits et activités censés porter l'avenir de l'entreprise.

Mais la formation intervient tardivement, une fois la crise sérieusement installée et après avoir été délaissée pendant des années. Un examen attentif des conditions d'accès à ces programmes et de leurs effets pour les salariés ne permet pas de conclure à un développement des capacités des personnes. Alors que l'accès à la formation pâtit d'un manque de transparence, d'une absence de procédures et de critères clairement établis, les programmes ne débouchent sur aucune certification susceptible d'assurer leur reconnaissance et leur transférabilité en cas de fermeture, de licenciement ou de départ volontaire.

Prenons l'exemple de Matrix. "Pour le nouvel atelier, on choisit les meilleurs », nous confiait le responsable de la production. Dans une usine où la population ouvrière est majoritairement féminine, avec quinze à vingt ans d'ancienneté, les «meilleurs» s'avéraient être des hommes, jeunes et relativement qualifiés. Le premier groupe envoyé en formation fut donc composé exclusivement de jeunes ouvriers, masculins et diplômés. Après avoir été embauchés comme pilotes de ligne $^{10}$, les jeunes ouvriers titulaires d'un bac, voire d'un bac +2 , se retrouvaient, à l'issue de la formation, assis sur une chaise à regarder au travers d'une loupe binoculaire pour effectuer des opérations extrêmement minutieuses ne nécessitant pas un niveau de formation supérieur au CAP (certificat d'aptitude professionnelle). Quelques semaines et grincements de dents plus tard, les «meilleurs » étaient devenus des femmes, le montage-câblage étant subitement devenu un travail

\footnotetext{
${ }^{10}$ Chez Matrix, les pilotes de ligne sont responsables de lignes de production entièrement automatisées. Ils surveillent les machines et coordonnent le travail d'ouvriers spécialisés chargés d'approvisionner les lignes en matières premières. Ils interviennent en cas de dysfonctionnement. Ce travail offre une grande autonomie et une liberté de mouvement.
}

spécifiquement féminin. En conséquence, le second groupe qui partit en formation ne comprenait que des ouvrières, jeunes de préférence.

Dans les entreprises en restructuration, le choix des personnes à former ne repose ni sur le niveau de qualification nécessaire pour effectuer un travail spécifique, ni sur la volonté d'offrir de nouveaux atouts à la frange la plus fragile du personnel. Selon ces deux critères, chez Matrix, le choix aurait dû porter sur les ouvrières embauchées de longue date, mais il a été motivé par la volonté de garder les individus identifiés comme potentiellement les plus employables et adaptables, capables de faire face à un environnement changeant. L'âge et le niveau de qualification initial sanctionné par un diplôme au moins équivalent au bac sont considérés comme les garants de ce potentiel d'adaptabilité. Nullement orientée vers le développement professionnel des personnes, la formation adossée à la restructuration impulse alors des parcours descendants, suscitant frustration et sentiment de déclassement.

D'ailleurs, pour la plupart des personnes rencontrées, l'entrée en formation ne représente pas un choix réel, comme le souligne cet ouvrier de Watson :

«J'étais à la maintenance [...] on m'a dit que je n'étais plus utile. Je me suis retrouvé sur la chaîne pendant au moins deux ans. Et puis on m'a dit soit tu vas en formation, soit tu restes sur la chaîne, soit tu pars. J'ai choisi la formation.» (Wat-10, ouvrier, 35 ans, 15 ans d'ancienneté, entré chez Watson juste après son service militaire, BEP d'opérateur-régleur sur systèmes d'usinage)

$\mathrm{Au}$ regard de ses qualifications initiales et des perspectives qui s'offrent à lui - un déclassement durable ou un licenciement dans une zone d'emploi déjà fortement touchée par les fermetures d'usine-, la formation constitue in fine la seule option sérieuse.

Toutefois, face à l'absence de certification de la formation, certains salariés s'interrogent sur sa finalité réelle: doter les salariés d'atouts de développement professionnel? Lancer une nouvelle activité ? Ou tout simplement calmer les esprits et préparer la fermeture ? Comme le suggère un responsable des ressources humaines :

« Je connais plein d'entreprises qui investissent dans la formation avant de fermer, c'est une façon d'acheter la paix sociale, je l'ai moi-même recommandé. » (Her-10, 
DRH, la cinquantaine, recruté un an avant l'entretien dans un contexte très perturbé, a été consultant pendant 10 ans, spécialiste des fermetures d'usines et des procédures d'outplacement)

Cet exemple attire l'attention sur la diversité des usages de la formation par les entreprises. Certes, les programmes de formation permettent aussi de préparer certaines personnes à une reconversion, mais les salariés n'ont que rarement l'opportunité d'exprimer leurs souhaits et de les faire valoir (Mazade, 2004 ; Leconte, 2002). Pourtant, la question du choix apparait primordiale pour toute politique de formation qui se veut aussi une politique de capacités.

Dans les entreprises en restructuration, les personnes ne disposent d'aucune latitude d'action. Le profil type de l'individu employable et adaptable dessiné par les directions repose essentiellement sur l'âge et le niveau scolaire, c'est-à-dire sur des ressources externes au contexte de travail, sur lesquelles l'individu ne peut plus rien. Seules les personnes dotées d'un capital scolaire, celles qui ont eu l'occasion de nouer des contacts professionnels à l'extérieur ou celles qui avaient déjà élaboré un projet professionnel alternatif peuvent trouver l'opportunité d'un nouveau départ. Cette configuration combinant absence d'opportunités et insécurité est la plus extrême que nous ayons rencontrée, les autres contextes organisationnels offrant, à défaut d'opportunités, une certaine sécurité.

\section{Avoir un travail dans une PME et le garder}

La taille des entreprises est une autre variable importante en matière de développement des capacités. Comme l'illustre le cas de Paintings, une taille critique suffisante et l'existence d'un marché interne du travail autorisant mobilité professionnelle et évolution de carrière, importent autant que la stabilité et la bonne santé économique. Paintings est un site de production de 30 personnes, dans le Choletais, d'une entreprise familiale de peintures et vernis établie à Lyon qui compte 120 salariés au total. À l'atelier, où travaille la grande majorité des salariés, aucune qualification spécifique n'est exigée. Le BTS (brevet de technicien supérieur) est requis pour les deux techniciens du laboratoire de contrôle, mais pour les ouvriers, le diplôme ne compte pas. Le profil recherché est celui d' " un gars qui est travailleur, qui en veut » (Paint-2, chef d'atelier, 35 ans, entré dans l'entreprise à 20 ans comme ouvrier avec un CAP de mécanique auto, promu 11 ans plus tard après avoir occupé, à sa demande, tous les postes d'opérateurs et de magasinier). La sélection passe par la mise à l'épreuve dans le cadre d'un contrat de travail intérimaire révocable à tout moment. Un second critère, non pas d'ordre professionnel mais personnel, rend en outre l'accès aux opportunités de recrutement extrêmement inégalitaire : "avoir 30 ans, un enfant, une maison et des dettes sur le dos» (Paint-1, responsable du site, 55 ans, entré dans l'entreprise en 1973 au laboratoire de contrôle avec un DUT de chimie). Ce critère vise avant tout la stabilité, avec un préjugé extrêmement défavorable pour les jeunes qui «ne tiennent pas » et par conséquent la négation de leur capacité à être recrutés.

Pour les salariés en poste, le nivellement des fonctions offre peu d'opportunités d'évolution. Le site compte un responsable, deux techniciens de laboratoire, une secrétaire, un chef d'atelier (depuis 2000) et les ouvriers, sans échelon intermédiaire. À l'exception du chef d'atelier, entré par intérim au conditionnement en 1988 et qui a, d'une certaine façon, créé son propre poste en montrant au responsable du site ce qu'un chef d'atelier pourrait lui apporter, les parcours ascendants sont structurellement improbables. Les ouvriers peuvent passer du broyage à l'empâtage ou au conditionnement, mais sans incidence sur leur classification ou leur salaire. Pourtant, le turnover est extrêmement faible: les ouvriers restent, même si les conditions de travail sont pénibles et les possibilités d'évolution extrêmement réduites. Pour les chimistes du laboratoire de contrôle, c'est l'inverse. Titulaires d'un diplôme qu'ils peuvent valoriser ailleurs, après trois ou quatre ans, ils rejoignent d'autres entreprises, faute de possibilité de promotion. Dans le cas de Paintings, évolution et promotion riment avec mobilité professionnelle externe. Mais encore faut-il avoir des atouts de mobilité et éventuellement être prêt à quitter «le pays » où l'emploi se fait rare, ce qu'excluent les ouvriers rencontrés. Déménager signifierait se couper des attaches personnelles et familiales, de formes d'entraide et de solidarité qui comptent autant que l'emploi. 
Chez Paintings, les discours se structurent autour du vouloir et du pouvoir - « celui qui veut (travailler) il peut $»-$, mais aucunement du choix et de la liberté. De choix, les ouvriers n'en ont pas. Comme le relève l'un d'entre eux :

« Je n'ai pas de diplôme, alors je peux pas demander l'impossible. L'ambiance est bonne et mon salaire est meilleur qu'avant. J'espère que je pourrai rester ici jusqu'à la retraite. [...] Je n'ai pas vraiment le choix. Le travail n'est pas facile à trouver. Il y a les circonstances économiques... J'ai pris la première offre, je ne voulais pas rester au chômage. Mais je ne le regrette pas. Je le referais. Mon plus grand regret est de ne pas avoir de diplôme, mais maintenant il est trop tard. » (Paint-3, ouvrier 45 ans, sans diplôme, a travaillé pendant 29 ans dans une entreprise de chaussures avant d'être licencié et d'entrer chez Paintings)

Comme si la résignation et les regrets par rapport à une scolarité manquée anéantissaient par avance tout espace de possibles. Avoir un travail et le conserver est un enjeu à tel point vital et problématique dans une région comme le Choletais, qu'il semble en exclure tout autre type d'attente par rapport au travail.

\section{Opter pour la sécurité et devenir éboueur}

Dans les services municipaux de propreté urbaine, les opportunités de développement professionnel sont rares, mais la sécurité de l'emploi est grande. Devenir éboueur n'est pas un rêve d'enfant. Toutes les personnes rencontrées mettent en avant leur statut d'agent municipal et de fonctionnaire en déclarant travailler «à la Ville», sans jamais mentionner le service particulier auquel elles sont affectées ${ }^{11}$. Avoir un emploi stable et sûr, se protéger du risque de licenciement et toucher un salaire à échéances régulières sont les raisons invoquées pour le choix de ce statut. De telle sorte que la sécurité de l'emploi vient compenser la dévalorisation sociale du métier.

\footnotetext{
${ }^{11}$ Les développements proposés ici s'appuient sur le matériau empirique recueilli lors de l'enquête aux Services techniques de la propreté de Paris (STPP). Cependant, les conclusions valent également pour les autres services municipaux étudiés.
}

Deux modes d'accès au métier se dégagent. Soit « la Ville » est le premier et unique employeur et on y est entré très jeune parce qu'on $\mathrm{y}$ «connaissait quelqu'un » - père, oncle, frère, voisin... Soit « la Ville » intervient après un passage dans le privé, généralement dans des emplois précaires, dans de mauvaises conditions de travail ou des entreprises à l'avenir incertain. Alors, dans la plupart des cas, cette décision s'inscrit dans une stratégie de protection à la suite d'une expérience traumatique, comme en témoigne cet interlocuteur :

«J'ai eu un licenciement et j'ai dit: licenciement, plus jamais. Il faut que je rentre dans la fonction publique. J'ai fait la RATP (Régie autonome des transports parisiens), la Mairie de Paris, le musée du Louvre... je me suis dit, le premier qui répond j'y fonce. J'ai pas eu d'autre réponse. » (PP-18/5-2, éboueur, la quarantaine, 10 ans d'ancienneté, CAPBEP plomberie-sanitaire)

Quel que soit le parcours antérieur, l'accès à la fonction publique territoriale est présenté comme un facteur de sécurité et, en conséquence, de promotion sociale ${ }^{12}$.

En revanche, les possibilités d'évolution professionnelle sont limitées. La voie de l'ancienneté et du concours, voie républicaine censée garantir une égalité de traitement, permet à certains éboueurs de passer chef d'équipe du nettoiement (CEN). Cependant, lorsqu'il s'agit d'atteindre le stade supérieur et de passer contremaître, les échecs sont nombreux, malgré les formations de remise à niveau proposées. La mobilité professionnelle peut également emprunter la voie de la promotion « au choix ». Pour ce type de promotion, les critères formels sont peu sélectifs et les décisions de nomination n'ont pas à être motivées. Le soutien du supérieur hiérarchique est nécessaire pour accéder à un grade plus élevé. Toutefois, la qualité du travail réalisé n'est pas un critère déterminant, les appuis familiaux, amicaux et syndicaux comptent bien plus. Ainsi, celui qui souhaite échafauder un parcours dont le balai ou la benne à ordures ne soit pas le seul horizon, doit construire et entretenir son propre réseau,

\footnotetext{
${ }^{12}$ Cette étude empirique corrobore les conclusions de l'étude sur l'enquête « emploi 2001 » menée par S. Gollac, en particulier sur l'accès à la fonction publique comme "refuge contre la précarité » (2005, p. 60).
} 
rassembler les informations ainsi que les appuis nécessaires. Forts ou faibles (Granovetter, 1974), les liens pertinents se construisent et s'alimentent en dehors de la sphère professionnelle proprement dite. In fine, le parcours dépend du capital social que chaque individu réussit à accumuler et de son habileté à le valoriser dans le contexte professionnel. Au-delà des critères officiels censés garantir une stricte égalité de traitement, les possibilités réelles d'évolution reflètent l'inéquitable répartition des ressources individuelles en termes de compétences sociales, d'insertion dans des réseaux, dans un contexte où aucun support collectif également accessible à tous ne vient corriger ce déséquilibre.

Dans les trois cas de figure qui viennent d'être étudiés, les opportunités sont non seulement peu nombreuses mais l'accès en est fortement inégalitaire. Si la situation économique de l'entreprise, sa taille, son statut ont une incidence directe sur le spectre des opportunités disponibles, l'inégalité d'accès à ces opportunités doit aussi être imputée à une politique des ressources humaines qui n'offre aucun support collectif permettant de corriger ces inégalités.

\section{DES OPPORTUNITÉS : LOGIQUE DE COMPÉTENCES VERSUS LOGIQUE DE CAPACITÉS}

À la différence des précédents, les contextes organisationnels suivants ont en commun d'offrir des opportunités d'évolution professionnelle à leurs salariés. Mais l'assignation de la responsabilité en matière de développement professionnel les distingue. Dans le premier contexte, la responsabilité relève du salarié en vertu d'une conception instrumentale de l'employabilité et des compétences. Dans le deuxième contexte, en revanche, l'entreprise s'implique dans le développement professionnel de ses salariés, faisant de la promotion de leurs capacités une dimension à part entière de la politique des ressources humaines.

\section{Le salarié responsable de son employabilité}

Comme les organisations étudiées jusqu'à présent, Helios et $\mathrm{H} 2 \mathrm{O}$ renvoient le salarié à sa propre responsabilité en matière de développement professionnel. En l'absence de crise grave, elles lui fournissent néanmoins un marché du travail interne (plus ou moins large selon leur taille) au sein duquel il peut évoluer, s'il est à même de se saisir des opportunités existantes. Le cas de $\mathrm{H} 2 \mathrm{O}$, assembleur aéronautique qui s'oriente vers le design industriel et passe ainsi d'un modèle de production «marchand» vers un modèle de «l'innovation », est emblématique.

Chez H2O, le changement d'activité se traduit par une politique massive de recrutement: plusieurs centaines de jeunes ingénieurs issus des écoles les plus prestigieuses ont été embauchés depuis 2002.

«Nous avons besoin de personnes ouvertes technologiquement, au-delà de nos propres compétences. Nous ne sommes plus seulement un assembleur mais un architecte » $(\mathrm{H} 2 \mathrm{O}-3$, responsable des relations sociales, membre du comité de direction, a fait toute sa carrière dans le groupe auquel appartient $\mathrm{H} 2 \mathrm{O}$, 52 ans).

Pourtant, le passage au nouveau modèle de production ne suffit pas à justifier l'exigence d'un tel niveau de qualification. Comme l'admettent plusieurs de nos interlocuteurs, dont l'un des membres du comité de direction, sur de nombreux postes, de bons techniciens ou des ingénieurs formés dans des écoles moins prestigieuses seraient tout à fait à leur place. À l'instar des cas de restructurations, le capital scolaire est ici conçu comme une garantie d'adaptabilité et de flexibilité. En ciblant les meilleures écoles d'ingénieurs, $\mathrm{H} 2 \mathrm{O}$ cherche à recruter des collaborateurs capables de prendre en charge leur développement professionnel et leur éventuelle reconversion en cas de coup dur.

En matière de recrutement, une telle inflation du diplôme revient à nier les capacités des personnes moins diplômées. En matière d'évolution interne, elle signifie que le développement professionnel est laissé à l'initiative individuelle et à la discrétion de la hiérarchie. Aucun outil formel d'accompagnement des carrières (du type entretien annuel par exemple) n'est utilisé dans l'entreprise.

«Tout se décide autour d'un café, à la fin des réunions, sur les contacts personnels... » (H2O-5, diplômée d'une école de gestion, cadre, entrée chez H2O à la sortie de l'école, 43 ans). 
«C'est du copinage, du bouche à oreille. À l'époque, fallait savoir ce qui se passait, y avait de l'affichage mais ça se faisait quand on avait le candidat. Moins maintenant, depuis 7-10 ans, mais quand même le bouche à oreille, les connaissances... la personne qui part doit être remplacée ou apporter son remplaçant » (H2O-11, BEP de chaudronnier, cadre, entré dans l'entreprise en 1980 alors qu'il intervenait sur le site en tant qu'intérimaire pour un sous-traitant, 54 ans).

Ainsi l'accès aux opportunités d'évolution de carrière repose essentiellement sur la personne, sur son insertion dans des réseaux professionnels, son habileté à mobiliser des leviers informels et à démontrer ses qualités. D'ailleurs, les salariés de $\mathrm{H} 2 \mathrm{O}$ utilisent beaucoup la forme active à la première personne pour parler de leur parcours: "J'ai demandé un programme de formation qualifiante» (H2O-5, cf. supra), «J'ai demandé ce poste » (H2O-7, ingénieur, responsable du recrutement des cadres, membre du comité de direction, a fait toute sa carrière dans le groupe auquel appartient $\mathrm{H} 2 \mathrm{O}, 56$ ans), contrairement aux salariés des entreprises en restructuration dont le discours est dominé par la forme passive : «On $m^{\prime} a$ demandé de... », « on m'a proposé de... ».

Par le passé, cette situation laissait chez H2O d'importantes marges de manœuvre à des personnes relativement peu diplômées, mais bien insérées et ayant fait la preuve de leurs qualités professionnelles. Elle permettait la construction de parcours originaux, quoique dans des conditions fortement inégalitaires. Mais l'arrivée massive de jeunes ingénieurs très qualifiés modifie la donne. D'une part, elle bloque l'horizon des techniciens embauchés dans les années 80. D'autre part, bien qu'intégrée dans un grand groupe international, $\mathrm{H} 2 \mathrm{O}$ ne sera pas en mesure d'offrir à tous ces jeunes ingénieurs des perspectives à la hauteur de leurs ambitions.

Le cas H2O montre que l'existence d'un marché interne du travail offrant des opportunités d'évolution est une condition nécessaire, mais non suffisante, pour le développement des capacités des salariés. Ce développement requiert également l'existence de supports collectifs, accessibles à tous, permettant de corriger les inégalités individuelles d'accès aux opportunités et de soutenir la conversion des ressources disponibles en réalisations effectives.

\section{L'appui du collectif}

Si l'existence d'opportunités de développement professionnel dépend de la taille de l'entreprise, de sa spécialisation et de sa situation économique, seules les entreprises qui cultivent une conception du collectif fondée sur des valeurs autres que marchandes concilient, dans notre échantillon, opportunités et supports collectifs. Sur les onze entreprises ou services étudiés, seuls deux relèvent de ce cas de figure. L'appartenance à un collectif et l'adhésion à des valeurs communes occupent une place centrale dans la politique de capacités développée par ces entreprises. Mais les valeurs sur lesquelles s'appuie le collectif sont contrastées. Ainsi Luz (chimie, 200 salariés) développe un sens du collectif fondé sur la famille et la religion, assorti d'anti-syndicalisme et de néo-paternalisme, alors que Bigtrucks (métallurgie, 450 salariés) promeut un sens du collectif appuyé sur la loyauté et la reconnaissance, entretenu par des pratiques de management participatif. L'instillation d'un sentiment d'appartenance et la constitution du collectif opèrent par conséquent sur des bases différentes.

Luz est une entreprise du secteur de la chimie implantée au Havre depuis 1956. Les pratiques de recrutement jouent un rôle décisif dans la constitution d'un collectif que la direction veut placer sous le signe d'un commun attachement à la famille, à la religion et d'un rejet de tout engagement syndical dans un territoire pourtant massivement marqué par la culture communiste. Pour ce faire, l'entreprise accueille de nombreux stagiaires de différentes écoles de chimie, afin de tester l'orientation des futures recrues, avant même leur entrée sur le marché $\mathrm{du}$ travail. Elle constitue par ce canal un vivier de salariés potentiels, qualifiés et capables de s'intégrer dans l'entreprise, c'est-à-dire possédant « la fibre Luz ». Étrangère à l'innovation, à la protestation et à toute idée de rupture, la «fibre Luz» renvoie à la tradition et à la continuité (Luz-2, responsable du recrutement, non diplômé, a fait toute sa carrière chez Luz depuis la sortie de l'école, 51 ans). Le mariage, garant d'une capacité à s'engager à long terme, de fidélité, de bonne moralité et de respect des traditions, est particulièrement apprécié. Au moment du recrutement, la sélection porte sur des modes de vie et des opinions (politiques et religieuses) autant 
que sur des compétences professionnelles, contrevenant ainsi aux prescriptions de non-discrimination formulées par la convention collective de la chimie (Convention collective des industries chimiques et connexes, 1952, article 5).

La « fibre Luz » n'est pas seulement exigée des salariés, elle façonne aussi la politique managériale, fortement teintée de paternalisme. L'entreprise verse des salaires légèrement supérieurs aux niveaux stipulés par la convention collective. Elle est particulièrement attentive à la sécurité, allant jusqu'à recenser les «presque accidents », et offre d'importantes possibilités de promotion interne à ses salariés. Les cadres sont pour moitié détenteurs d'un diplôme bac +5 et pour moitié autodidactes. Ces derniers, souvent anciens ouvriers de production auxquels Luz a permis d'accéder à un fauteuil au comité de direction, constituent les meilleurs défenseurs de la « fibre Luz ».

Satisfaire, si possible, les aspirations salariales et professionnelles des salariés représente une garantie pour tenir les organisations syndicales à distance. Aussi la politique de formation et de promotion interne contribue-t-elle à fonder et protéger le collectif, tout en soutenant le développement des capacités professionnelles des salariés. Des outils d'évaluation facilement accessibles sur l'intranet, des critères clairement formulés visent une équité, au moins formelle, en ce domaine. Les salariés se sentent concernés par leur entreprise et manifestent un sentiment d'appartenance à son égard. Expression de cette socialisation partagée, le midi à la cantine après le repas, la majorité des salariés joue à la manille, tous niveaux hiérarchiques confondus. Mais «la fibre Luz » n'autorise pas une grande diversité d'opinions et de convictions, bien au contraire. Si la politique de Luz contribue au développement de la capacité à travailler, d'autres capacités de base y sont niées, comme la capacité à prendre la parole et à faire valoir une voix dissonante.

Chez Bigtrucks, assembleur de poids lourds, à l'inverse, la constitution du collectif s'appuie sur la participation et l'expression de la pluralité des opinions en présence. Elle est orchestrée par une politique de management participatif fondée sur le principe de «l'amélioration continue » et de groupes de travail associant des salariés de différents niveaux. Par ce biais, la direction cherche à motiver des salariés qui sont en grande majorité des opérateurs travaillant sur la ligne d'assemblage, en leur assurant reconnaissance et prise en compte, conformément à une conception selon laquelle la performance résulte de la conjonction entre motivation et compétences (Big-4, responsable ressources humaines, 40 ans, entré à 28 ans dans l'entreprise en tant que responsable qualité, puis responsable méthodes, avant de passer 9 ans plus tard à la GRH, diplôme bac +4 , passe un DESS - diplôme d'études supérieures spécialisées en formation continue au moment de l'entretien). Et de fait, la plupart des salariés insistent sur le fait qu'ils se sentent reconnus, qu'on leur fait confiance et qu'ils se trouvent bien dans l'entreprise.

L'entreprise constitue un collectif auquel les salariés s'identifient. Le produit, en l'occurrence le camion dont on est fier et à la production duquel tout le monde contribue, joue un rôle fédérateur. Le collectif de Bigtrucks se construit d'abord autour de l'image de son produit. Mais ce collectif est aussi le résultat d'un ensemble d'outils structurants accessibles et connus de tous, tels que les référentiels métiers ou « la maison Bigtrucks $»^{13}$ affichée dans chaque bureau, atelier et lieu de pause. Chez Bigtrucks, la direction ne parle pas de «fibre », mais d' « esprit» et dans «l'esprit Bigtrucks, les hommes et l'organisation forment un tout, il faut les penser ensemble » (Big-2, directeur ressources humaines, 56 ans, juriste, dans le groupe Bigtrucks-France depuis 25 ans, nommé DRH à l'ouverture du site en 1992 en plus de ses anciennes fonctions qu'il conserve dans le groupe). Le système Bigtrucks résulte d'une alchimie entre la formalisation par les outils - offrant autant de principes généraux et partagés d'action - et la valorisation de la dimension interactive du travail - jouant sur l'expression de la singularité et la prise de responsabilité de chacun. Ce système s'appuie sur un important travail de communication interne à, et entre, tous les niveaux : de la publication du Petit porteur, journal bimensuel, jusqu'aux groupes de travail mixtes qui permettent aux salariés de sortir de

\footnotetext{
${ }^{13}$ La « maison Bigtrucks » est un outil de management synthétisant les trois priorités de l'entreprise, symbolisées par les trois piliers de la politique de l'entreprise: "priorité au client", « respect des personnes», «éliminer le gaspillage». L'étage visualise les priorités dans le travail quotidien : «sécurité/environnement, qualité, délai de livraison, coût », alors que le toit est dédié au principe de «l'amélioration continue ».
} 
la ligne, pour des durées variables, afin de participer à l'amélioration continue sur le moyen terme, ou en temps réel en cas de problème ponctuel et urgent.

Dans notre échantillon, Bigtrucks est l'entreprise qui offre les conditions les plus favorables au développement des capacités des salariés. Et ce aussi bien en matière de recrutement (la sélection s'y opère par la mise à l'épreuve professionnelle sans que le jeune âge, l'absence de bac, les opinions religieuses ou politiques constituent des critères éliminatoires $a$ priori), que d'évolution interne. De nombreux chefs d'atelier sont des anciens opérateurs. Lorsqu'un poste se libère, il fait systématiquement l'objet d'un affichage interne. Mais le service des ressources humaines dispose aussi d'une "potential list », constituée avec l'aide des managers appelés à identifier les salariés chez lesquels ils perçoivent un potentiel d'évolution indépendamment de toute vacance de poste. Les efforts de formation portent par anticipation sur ces personnes.

Ainsi Olivier, 30 ans, est entré chez Bigtrucks en 1993, avec un CAP, comme magasinier. En quelques années, il a accédé à l'échelon de pilote (chef d'équipe), mais sa progression dans la classification de la métallurgie est dorénavant bloquée en raison de son niveau d'étude. Au moment de l'entretien, il s'apprête à commencer une formation de technicien d'une durée de huit mois (dans le cadre d'un congé individuel de formation), afin de pouvoir à son retour postuler sur un poste de chef d'atelier, s'il s'en libère un. Autre exemple, celui de François, 33 ans, qui est également entré avec un CAP (en 1992) et qui a depuis accédé à l'échelon de pilote, mais qui entreprend une formation pour se reconvertir dans la vente audio-visuelle et, à terme, quitter Bigtrucks. Tous deux soulignent l'importance du soutien et des encouragements de leur «(ma) responsable $R H$ ». Mais même chez Bigtrucks, qui réunit les conditions les plus favorables aux capacités que nous ayons pu observer, certains facteurs personnels limitent l'accès aux ressources: l'âge avancé (plus de 50 ans), le sexe (être une femme dans le milieu du poids lourd) et le militantisme syndical fonctionnent ici aussi comme des obstacles ou des ralentisseurs de parcours. En outre, l'enquête a révélé d'importantes inégalités dans la faculté des personnes à se saisir des opportunités et des supports collectifs offerts par l'organisation. Leur existence ne suffit pas, encore faut-il que le salarié se sente habilité à s'en saisir, à formuler un projet et à en défendre la légitimité auprès de ses supérieurs hiérarchiques. Toutes choses qui supposent des aptitudes à s'exprimer et s'imposer qui sont loin d'être également distribuées entre les personnes et les sexes dans un monde fortement marqué par le symbole de force et de virilité du poids lourd.

Le modèle de management participatif, tout comme celui des affinités religieuses et politiques, lie l'accès aux opportunités et aux supports collectifs à l'implication personnelle dans un collectif fortement structuré. Chez Bigtrucks et Luz, la direction essaie de développer les voies d'une prise de parole collective sous contrôle managérial, parallèlement aux canaux traditionnels de l'action syndicale. Très différentes quant à leurs fondements, ces initiatives satisfont des demandes de considération et de reconnaissance des salariés et engendrent des appréciations relativement favorables. Néanmoins, elles tendent à imposer un modèle où la participation devient une norme et où les comportements de retrait sont condamnés et constituent des facteurs de conversion négatifs ${ }^{14}$. Elles posent en outre la délicate question de l'articulation de ces formes de participation avec l'action collective des salariés, notamment syndicale.

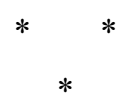

Pour conclure, on peut positionner les entreprises de notre échantillon dans le tableau 1.

En haut à gauche (--) du tableau se situent les entreprises en restructuration qui n'offrent ni opportunités ni sécurité aux salariés. Elles sont gouvernées par un management de crise qui ne fait guère de place à une politique de développement des capacités, en dépit des prescriptions formelles du type plans sociaux. Cette première configuration se caractérise par

\footnotetext{
${ }^{14}$ L'opération de conversion permet la transformation des ressources en réalisations effectives. Cette distinction entre ressources et réalisations fait des facteurs de conversion des éléments décisifs qui différencient l'approche par les capacités des théories de la justice fondées sur les ressources, comme celle de J. Rawls (Robeyns 2005, p. 99). D'ordre personnel, social ou environnemental, les facteurs de conversion dépendent à la fois des personnes, des organisations, des institutions et des situations concrètes d'action.
} 
Tableau 1

Les capacités : une conjonction entre opportunités et sécurité

\begin{tabular}{|c|c|c|c|}
\hline & & \multicolumn{2}{|c|}{ Sécurité de l'emploi } \\
\hline & & - & + \\
\hline \multirow{2}{*}{$\begin{array}{c}\text { Opportunités } \\
\text { de développement }\end{array}$} & - & $\begin{array}{c}-- \\
\text { Entreprises en restructuration } \\
\text { Supports collectifs + mais non orientés } \\
\text { vers les capacités } \\
\text { Management de crise }\end{array}$ & $\begin{array}{c}-+ \\
\text { Services municipaux, Paintings } \\
\text { Supports collectifs + mais non orientés } \\
\text { vers les capacités } \\
\text { Ressources personnelles }\end{array}$ \\
\hline & + & $\begin{array}{c}\mathrm{H} 2 \mathrm{O}, \text { Hélios } \\
\text { (moyennes et grandes entreprises) } \\
\text { Supports collectifs - } \\
\text { Ressources personnelles }\end{array}$ & $\begin{array}{c}++ \\
\text { Luz, Bigtrucks } \\
\text { (moyennes et grandes entreprises) } \\
\text { Supports collectifs }+ \\
\text { Management fondé sur des valeurs }\end{array}$ \\
\hline
\end{tabular}

l'absence de liberté de choix et de pouvoir d'agir pour le salarié. La deuxième configuration en haut à droite $(-+)$ du tableau associe une latitude de choix extrêmement limitée à une sécurité relative de l'emploi. Elle correspond aux services municipaux de propreté urbaine où le statut assure la sécurité mais rend l'accès aux rares opportunités de développement professionnel fortement inégal. Elle correspond encore à des entreprises privées comme Paintings, en bonne santé économique mais où les opportunités de développement sont rares. La petite taille n'est pas un facteur exclusif de cette rareté qui peut être liée dans des plus grandes entreprises au type de management, privilégiant par exemple le recrutement externe à la mobilité interne. Quelle que soit la raison de ce défaut d'opportunités, l'évolution professionnelle relève dans cette configuration à titre principal de ressources personnelles et relationnelles (connaissances interpersonnelles, savoir s'imposer...) inégalement distribuées. La même prégnance d'une culture de l'arrangement interpersonnel vaut pour la troisième configuration $(+-)$. Cette dernière rassemble des entreprises qui, de par leur taille notamment, offrent des opportunités de développement mais peu de supports collectifs, laissant aux salariés l'entière responsabilité de leur parcours professionnel et l'incertitude de son évolution. Enfin, la dernière configuration $(++)$ regroupe des entreprises comme Luz ou Bigtrucks qui associent liberté de choix et pouvoir d'agir. Le développement professionnel y relève d'une responsabilité collective fondée sur un management par les valeurs. Seule cette dernière configuration se révèle favorable, avec toutefois les importantes limites que nous avons pointées, au déploiement des capacités des salariés.

L'échantillon est trop limité pour déboucher sur une typologie raisonnée croisant de manière systématique taille, secteur, produit, type de management et formes collectives de l'organisation, d'autant plus que ces différentes variables apparaissent plus ou moins discriminantes et pertinentes en fonction des configurations. Ainsi, une très petite taille est corrélée à un manque d'opportunités et par conséquent de liberté de choix, mais à l'inverse une taille plus grande ne garantit pas l'existence d'opportunités et encore moins le pouvoir d'action des salariés. Celui-ci est tributaire d'autres variables telles que le type de management, le degré et la qualité de l'organisation collective au sein de l'entreprise. Ainsi chez Luz et Bigtrucks, la taille ou le modèle de production ne sont pas des facteurs déterminants pour le développement d'une politique de capacités. Au-delà de la bonne santé économique de l'entreprise, l'essentiel repose sur la conception des supports collectifs et les valeurs qui les portent. Si le management, en tant que pratique formalisée de gouvernement des organisations, apparaît comme un facteur discriminant pour les deux configurations extrêmes $(--)$ et $(++)$, il est moins décisif dans les configurations intermédiaires $(-+)$ et $(+-)$ 
davantage marquées par les ressources d'une culture de l'arrangement interpersonnel. Enfin, caractéristiques de la dernière configuration $(++)$ favorable aux capacités, les supports collectifs apportés par l'entreprise sont très inégaux dans les autres cas. Ils ne se caractérisent pas forcément par leur absence comme dans la configuration $(+-)$, ils peuvent aussi exister dans une forme qui se révèle inappropriée à soutenir le développement des capacités comme dans les services municipaux de propreté ou dans certains contextes de restructuration. Les services municipaux de propreté montrent en outre clairement que la seule sécurité de l'emploi ne suffit pas à promouvoir les capacités des salariés.

Comme l'illustrent les cas de Luz et Bigtrucks, le développement des capacités en entreprises suppose l'existence de supports collectifs ciblés afin de corriger les inégalités d'accès aux opportunités. Ces supports peuvent prendre la forme de politiques de formation, de soutien et d'accompagnement des projets individuels d'évolution professionnelle, mais également d'accès à l'information et de dispositifs de participation. En d'autres termes, le développement des capacités suppose que soit accordée une valeur spécifique à l'humain et cultivé un sens du collectif favorable à son développement. Mais comme l'illustre Luz, la participation et le sens du collectif ne riment pas forcément avec l'expression et la représentation de la pluralité des opinions, pourtant requises pour toute politique de capacités.

Chez Luz, le caractère fortement sélectif des valeurs sur lesquelles se fonde le collectif limite l'accès aux capacités aux seuls salariés qui ont réussi à franchir des pratiques de recrutement fortement discriminatoires, voire illégales. Chez Bigtrucks en revanche, les valeurs se veulent plurielles et le collectif est associé à l'ouverture d'un espace critique sur le travail, notamment à travers le processus d'amélioration continue et les groupes de travail. En d'autres termes, les capacités professionnelles des salariés ne sont pas uniquement liées à leurs compétences, mais pour une large part aussi à leur capacité d'expression, de participation et d'accès à l'information. Elles requièrent non seulement des opportunités et des supports collectifs, mais également la libre expression des salariés dans une entreprise conçue comme organisation pluraliste. Parce que, comme le rappelle I. Berlin (1988, p. 218), l'idéal de liberté de choix entre diverses fins implique un certain pluralisme des valeurs, les modalités de participation et d'association des salariés à la vie de l'entreprise occupent une place centrale dans toute politique de capacités.

\section{Bibliographie}

Barbier J.-C. (2002), « Peut-on parler d'activation de la protection sociale en Europe ? », Revue française de sociologie, 43 (2), pp. 307-332.

Berlin I. (1988) (1969), Éloge de la liberté, Paris, Calmann-Lévy.

Boltanski L., Thévenot L. (1991), De la justification. Les économies de la grandeur, Paris, Gallimard.

Bureau M.-C., Marchal E. (éds) (2005), Au risque de l'évaluation : salariés et candidats soumis aux aléas du jugement, Villeneuve d'Ascq, Presses Universitaires du Septentrion.
Castel R., Haroche C. (2000), Propriété privée, propriété sociale, propriété de soi, Paris, Fayard.

Commission européenne (2000), Directives pour l'emploi pour l'année 2001, COM, 548 final.

Convention collective nationale des industries chimiques et connexes, 30 décembre 1952, www.legifrance.fr

Corteel D., Zimmermann B. (2006), Employability, Voice and Security: An Inquiry into the Capabilities of Workers in French Firms, Paris, Document de Travail de l'IDHE, $\mathrm{n}^{\circ}$ 06-01. 
Dejours C. (2003), L'évaluation du travail à l'épreuve du réel. Critique des fondements de l'évaluation, Paris, INRA Éditions.

Eymard-Duverney F., Marchal E. (1997), Façons de recruter : le jugement des compétences sur le marché du travail, Paris, Métailié.

Gasper D. (2002), « Is Sen's capability approach an adequate basis for considering human development? », Review of Political Economy, 14 (4), pp. 435- 461.

Gollac S. (2005), «La fonction publique: une voie de promotion sociale pour les enfants des classes populaires? Une exploitation de l'enquête "emploi 2001" ", Sociétés contemporaines, $\mathrm{n}^{\circ} 58$, pp. 41-64.

Granovetter M. (1974), Getting a job : A Study of Contacts and Careers, Harvard University Press, Cambridge Mass.

Leconte G. (2002), « De la mine au chantier, conversion professionnelle d'anciens mineurs/futurs maçons », Formation Emploi, $\mathrm{n}^{\circ}$ 78, avril-juin, pp. 69-77.

Mazade O. (2004), «Passé professionnel et reconversions : le reclassement des mineurs du NordPas-De-Calais, Formation Emploi, ${ }^{\circ}$ 85, janviermars, pp. 41-55.
Passeron J.-C. (1990), « Biographies, flux, itinéraires, trajectoires", Revue Française de Sociologie, XXXI, pp. 3-22.

Robeyns I. (2005), « The capability approach: a theoretical survey », Journal of Human Development, 6 (1), pp. 93-114.

Salais R., Storper M. (1993), Les mondes de production : enquête sur l'identité économique de la France, Paris, Éditions de l'EHESS.

Sen A. (2003) (1990), «La liberté individuelle : une responsabilité sociale", in L'économie est une science morale, Paris, La Découverte, pp. 43-76.

Zalio P.-P., Fouilland B. (éds), (2004), Cultures entrepreneuriales et gouvernance locale et développement économique, rapport de recherche, appel à proposition du GIS IRSIT 2002-2003 « La problématique du développement au Havre», contrat de recherche RO 425.

Zimmermann B. (2006a), «Employabilité et logique de compétences », in R. Salais et R. Villeneuve (éds.), Développer les capacités des hommes et des territoires en Europe, Paris, Éditions de l'Anact, pp. 74-93.

Zimmermann B. (2006b), «Pragmatism and the capability approach. Challenges in social theory and empirical research », European Journal of Social Theory, 9 (4), pp. 467-484.

\section{Résumé}

\section{Capacités et développement professionnel}

\section{Delphine Corteel et Bénédicte Zimmermann}

Comment s'articulent opportunités sociales, supports collectifs et latitude d'action individuelle dans le développement professionnel des salariés en entreprise ? L'approche par les capacités d'A. Sen est ici mobilisée dans le cadre d'une enquête menée auprès de huit entreprises françaises de la chimie, de la métallurgie et de trois services municipaux de propreté urbaine. Sont étudiés les moments charnières au cours desquels les capacités sont mises à l'épreuve et rendues visibles (recrutement, formation, mobilité professionnelle) ainsi que les critères d'évaluation qui président à ces moments. Plusieurs configurations organisationnelles se distinguent alors par la façon dont elles articulent opportunités de développement et supports collectifs.

\section{Mots-clés}

Approche par les capabilités/capacités, employabilité, cheminement professionnel, enquête, entreprise, France Journal of Economic Literature: D 63, J 24, J 59 


\section{Horiznns}

$\mathbf{N}^{\circ} 4$ - avril 2007

\section{DOSSIER}

\section{Les jeunes dans une société vieillissante}

\section{ANALYSE}

- Autonomie et insertion des jeunes adultes, une comparaison FranceDanemark (Cécile Van de Velde)

- Le passage tardif à l'âge adulte des Italiens : entre maintien

du modèle traditionnel et individualisation

des trajectoires biographiques (Vincenzo Cicchelli et Maurizio Merico)

- Entre autonomie et insertion. Les grands dispositifs de la politique

de la jeunesse en Allemagne (Monika Salzbrunn)

- L'évolution des valeurs des jeunes (Régis Bigot)

\section{PISTES POUR L'ACTION}

- Introduction à l'analyse du BEPA (Julien Damon)

- Investing in Youth : from childhood to adulthood (BEPA)

\section{DÉBAT}

- "Qu'en est-il des rapports intergénérationnels en France ? " avec Louis Chauvel, professeur à Sciences-Po, Jean-Philippe Viriot-Durandal, MC en sociologie, auteur du Pouvoir gris, et André Masson, CNRS-Delta

\section{FOCUS}

- L’Assemblée nationale est-elle trop âgée ? (Gabriel Arnoux)

\section{VARIA}

- L'influence française au sein du Parlement européen à mi-mandat. Quelle stratégie à l'horizon 2009 ? (Clément Beaune et Thierry Chopin)

- Concurrence et innovation : quelles politiques pour favoriser le développement des entreprises?

(Rémi Lallement et Vanessa Wisnia-Weill)

- Les politiques familiales dans l'Union européenne et la Stratégie de Lisbonne : quelques enseignements de l'expérience française (Pierre Courtioux et Olivier Thevenon)

- Accords et désaccords sur l'avenir de l'Union européenne (Marie-Cécile Milliat)

\section{CHRONIQUES}

- Présentation d'un homologue étranger : La section Planification et stratégie de la Chancellerie fédérale suisse (Jean-François Le Bihan et Aude Rousselot)

- Actualité des homologues étrangers (Aude Rousselot)

- Échos (Colloques et Comptes rendus de lecture ; Analyses critiques)

- Actualités du Centre d'analyse stratégique 